\title{
Derivative of Unitary is not always -iHU
}

\author{
A S Muddu ${ }^{1}$ \\ ${ }^{1}$ Reliance Corporate Park, Reliance Industries Limited, Mumbai 400701, India
}

December 23, 2020

\begin{abstract}
$\$ \mathrm{i} \backslash \operatorname{dot}\{\mathrm{U}\}=\mathrm{HU} \$$ (or $\$ \backslash \operatorname{dot}\{\mathrm{U}\}=-\mathrm{iHU} \$$ ) is the equation that is said to govern the evolution of a unitary matrix $\$ \mathrm{U} \$$ given the Hamiltonian $\$ \mathrm{H} \$$ of the system. This equation is said to hold true even if the Hamiltonian is time dependent. We show iU = HU (or $\$ \backslash \operatorname{dot}\{\mathrm{U}\}=-\mathrm{iHU} \$$ ) is the equation that is said to govern the evolution of a unitary matrix $\$ \mathrm{U} \$$ given the Hamiltonian $\$ H \$$ of the system. This equation is said to hold true even if the Hamiltonian is time dependent. We show in this paper that $\$ i \backslash \operatorname{dot}\{\mathrm{U}\}=\mathrm{HU} \$$ may not always hold for time dependent Hamiltonians.this paper that $\$ i \backslash \operatorname{dot}\{U\}=\operatorname{HU} \$$ may not always hold for time dependent Hamiltonians.
\end{abstract}

\section{Introduction}

The evolution of a unitary operator is given by $i \dot{U}=H U$. (where $H$ is the Hamiltonian of the system). This equation is so common place that no one bothers to cite it. It is considered as a standard part of the curricula of certain courses, in physics books (see [1]) and even in allied engineering fields. For example see [2] ( a course in Nuclear Engineering). Needless to say, the formula also has been extensively used in the literature. For example see Equation 35 in [3], Equation 25 in [4], Equation 7 in [5], Equation 4 in [6].

The organisation of next section is as follows:

In subsection $\mathrm{A}$ we show that for any matrix $A,[A, \dot{A}]$ is not necessarily equal to $\mathbf{0}$. Subsection $\mathrm{B}$ deals with the derivative of $e^{A}$. From this we find the derivative of a unitary matrix in subsection $\mathrm{C}$. We show in subsection D as to why in case of time independent Hamiltonian $i \dot{U}=H U$ more or less holds good.

Finally in section III, we synthesize the various lines of argument into a concluding paragraph.

\section{Proof}

\section{A and its time derivative don't commute}

The title can be mathematically paraphrased as 
$[A, \dot{A}] \neq \mathbf{0}$. For any function $f(A)$ (matrix or scalar valued) $\dot{f}=\frac{\partial f}{\partial t}$. Say we have a matrix valued function

$$
A(t)=\left[\begin{array}{ll}
a & b \\
c & d
\end{array}\right]
$$

where a,b,c,d are four distinct functions of time. We can differentiate $A(t)$ as follows [7], [8]

$$
\dot{A}=\left[\begin{array}{ll}
\dot{a} & \dot{b} \\
\dot{c} & \dot{d}
\end{array}\right]
$$

Thus,

$$
\begin{aligned}
{[A, \dot{A}] } & =A \dot{A}-\dot{A} A \\
& =\left[\begin{array}{ll}
a & b \\
c & d
\end{array}\right]\left[\begin{array}{cc}
\dot{a} & \dot{b} \\
\dot{c} & \dot{d}
\end{array}\right]-\left[\begin{array}{cc}
\dot{a} & \dot{b} \\
\dot{c} & \dot{d}
\end{array}\right]\left[\begin{array}{cc}
a & b \\
c & d
\end{array}\right] \\
& =\left[\begin{array}{cc}
b \dot{c}-\dot{b} c & a \dot{b}-\dot{a} b+b \dot{d}-\dot{b} d \\
c \dot{a}-\dot{c} a+d \dot{c}-\dot{d} c & -(b \dot{c}-\dot{b} c)
\end{array}\right]
\end{aligned}
$$

Any of the elements of $[A, \dot{A}]$ are not necessarily zero at all times. Thus $[A, \dot{A}]$ is not necessarily zero. Special cases where $[A, \dot{A}]=\mathbf{0}$

1. A is a constant function. This makes $\dot{A}=\mathbf{0}$. Because of this $[A, \dot{A}]=\mathbf{0}$

2. $A=\lambda(t) I \Rightarrow \dot{A}=\dot{\lambda}(t) I$

$$
\begin{aligned}
\therefore[A, \dot{A}] & =A \dot{A}-\dot{A} A \\
& =(\lambda I)(\dot{\lambda} I)-(\dot{\lambda} I)(\lambda I) \\
& =(\lambda \dot{\lambda} I)-(\dot{\lambda} \lambda I) \\
& =\mathbf{0}
\end{aligned}
$$

3. $A$ is a diagonal matrix. This implies that $b, c=0$. From Equation 3 we have $[A, \dot{A}]=\mathbf{0}$.

4. $A=t B$, where $B$ is a constant matrix.

$$
\begin{aligned}
\therefore[A, \dot{A}] & =A \dot{A}-\dot{A} A \\
& =t B B-B t B \\
& =\mathbf{0}
\end{aligned}
$$

It can be easily shown that even if $\mathrm{A}$ is a skew Hermitian $[A, \dot{A}]$ is not necessarily zero. For the rest of discussion we only consider A such that $[A, \dot{A}] \neq \mathbf{0} \forall t$

In the case $[A, \dot{A}]=\mathbf{0}$, everything is true and wonderful. 


\section{Derivative of matrix exponential functions}

Let us see what we get on differentiating $A^{2}$. From the product rule, we have

$$
\left(\dot{A}^{2}\right)=A \dot{A}+\dot{A} A
$$

But, given the previous discussion

$$
\begin{aligned}
\left(\dot{A}^{2}\right) & \neq \dot{A} A+\dot{A} A(\because[A, \dot{A}] \neq 0) \\
& \neq 2 \dot{A} A
\end{aligned}
$$

Similarly,

$$
\left(\dot{A^{3}}\right) \neq 3 \dot{A} A
$$

Instead, we obtain

$$
\left(\dot{A}^{3}\right)=A A \dot{A}+A \dot{A} A+\dot{A} A A
$$

For $A^{n}$, we get

$$
\begin{aligned}
\left(\dot{A^{n}}\right) & =\left(A \dot{A^{n-1}}\right) \\
& =A\left(A^{\dot{n}-1}\right)+\dot{A} A^{n-1}
\end{aligned}
$$

Continuing down, we are left with

$$
\left(\dot{A^{n}}\right)=\sum_{m=0}^{n-1} A^{m} \dot{A} A^{n-(m+1)}
$$

We know that

$$
e^{A}=\sum_{n=0}^{\infty} \frac{A^{n}}{n !}
$$

On applying the above train of thought

$$
(\dot{e} A)=\sum_{n=0}^{\infty} \sum_{m=0}^{n-1} \frac{A^{m} \dot{A} A^{n-(m+1)}}{n !}
$$

[9] and [10] have rewritten the above formula in a more pleasing format. The simplified form of Equation 2.1 from [10] in terms of our notation is as follows

$$
\left(\dot{e^{A}}\right)=\int_{0}^{1} e^{A(1-s)} \dot{A} e^{A s} d s
$$

Let us try to derive Equation 15 from Equation 14. The steps below are inspired from [9].

When we differentiate $A^{n}$ we obtain a series in which each term is a permutation of a product of $n-1 A^{\prime} s$ and one $\dot{A}$. So the series in Equation 14 can be rewritten as: 


$$
\begin{aligned}
(\dot{e} A) & =\sum_{p=0}^{\infty} \sum_{m=0}^{\infty} \frac{A^{m} \dot{A} A^{p}}{(m+p+1) !}(\because n=m+p+1) \\
& =\sum_{p=0}^{\infty} \sum_{m=0}^{\infty} \frac{A^{m} \dot{A} A^{p}}{m ! p !} \frac{m ! p !}{(m+p+1) !}
\end{aligned}
$$

We know that

$$
\int_{0}^{1}(1-s)^{m} s^{p} d s=\frac{m ! p !}{(m+p+1) !}
$$

Hence:

$$
\begin{aligned}
\left(\dot{e}^{A}\right) & =\sum_{p=0}^{\infty} \sum_{m=0}^{\infty} \frac{A^{m} \dot{A} A^{p}}{m ! p !} \int_{0}^{1}(1-s)^{m} s^{p} d s \\
(\dot{e} A & =\int_{0}^{1} \sum_{p=0}^{\infty} \sum_{m=0}^{\infty} \frac{(1-s)^{m} A^{m} \dot{A} A^{p} s^{p}}{m ! p !} d s \\
(\dot{e} A) & =\int_{0}^{1} \sum_{p=0}^{\infty}\left(\sum_{m=0}^{\infty} \frac{(1-s)^{m} A^{m}}{m !}\right) \dot{A} \frac{A^{p} s^{p}}{p !} d s \\
(\dot{e} A & =\int_{0}^{1} \sum_{p=0}^{\infty} e^{(1-s) A} \dot{A} \frac{A^{p} s^{p}}{p !} d s \\
\left(\dot{e^{A}}\right) & =\int_{0}^{1} e^{(1-s) A} \dot{A} e^{A s} d s
\end{aligned}
$$

Since, $[A, \dot{A}] \neq \mathbf{0},\left[e^{A}, \dot{A}\right] \neq \mathbf{0}$. Given that $\left[e^{A}, \dot{A}\right] \neq \mathbf{0}$, we can say that

$$
\begin{aligned}
e^{A(1-s)} \dot{A} e^{A s} & \neq \dot{A} e^{A(1-s)} e^{A s} \\
& \neq \dot{A} e^{A}
\end{aligned}
$$

Hence,

$$
\begin{aligned}
& \left(\dot{e^{A}}\right) \neq \int_{0}^{1} \dot{A} e^{A} d s \\
& \left(\dot{e^{A}}\right) \neq \dot{A} e^{A}
\end{aligned}
$$

From Equation 15, 24 and 25 one can safely say that $\left(e^{\dot{A}}\right)$ can not be written as $B e^{A}$ (where $B=g(\dot{A})$ i.e B is a function of only $\dot{A}$ ).

\section{Derivative of a unitary matrix}

We can write $U=e^{\Omega(t)}$, where $\Omega(t)$ is a matrix valued function of time. By the virtue of its construction, $\Omega(t)$ is an skew Hermitian matrix. Thus from end of section, $[\Omega, \dot{\Omega}] \neq \mathbf{0}$ 
Since $\left(e^{\dot{A}}\right) \neq B(\dot{A}) e^{A}$ as proved in end of section

$$
\begin{aligned}
& \dot{U} \neq B(\dot{\Omega}) e^{\Omega} \quad \text { or } \\
& \dot{U} \neq B(\dot{\Omega}) U
\end{aligned}
$$

In words it means that $\dot{U}$ cannot be written as a product of function of $B(\dot{\Omega})$ and $U$. We know that for time independent Hamiltonians $U=e^{-i H t}$. So it is fair to assume that $\Omega$ may depend on the Hamiltonian $H$ in some way. Since $\Omega$ depends on the Hamiltonian $H, \dot{\Omega}$ too is a function of $H$.

$\therefore$ From the previous Equation 26

$$
\dot{U} \neq B^{\prime}(H) U
$$

where $B^{\prime}$ is another function only of $H$ such that $B^{\prime}(H)=B(\dot{\Omega})$.

Taking things a step further $\dot{U} \neq-i H U$ for time dependent Hamiltonians.

\section{Time independent Hamiltonians}

The rule $\dot{U}=-i H U$ still holds good for time independent Hamiltonians, but here too things are not the same as before. From special case 1 from subsection we have

$$
[H, \dot{H}]=\mathbf{0} \quad \text { since } H \text { is time independent }
$$

From the discussion in sub-section we can say that

$$
\begin{aligned}
{\left[H, e^{-i H t}\right] } & =\mathbf{0} \\
{[H, U] } & =\mathbf{0}
\end{aligned}
$$

Thus $\dot{U}=-i H U$ can be transformed to $\dot{U}=-i U H$. So The order of $U, H$ does not really matter on the right hand side the equation $\dot{U}=-i H U$ for time independent Hamiltonians.

\section{Conclusion}

In this paper, we have shown that $\dot{U}$ is not always equal to $-i H U$ for time dependant Hamiltonians. This does not mean that it is not possible. One of the ways it may be possible is that the functions $a, b, c$, $d$ of $A$ align themselves in such a way that $[A, \dot{A}]=\mathbf{0}$ (other than those special cases considered in section ). Under more severely restrictive conditions than those considered here, [12], [13] have shown that $(\dot{e} A)=\dot{A} e^{A}$, even if $[A, \dot{A}] \neq \mathbf{0}$. But these restrictions coupled with the Hermiticity requirements of the Hamiltonian make this very unlikely to happen.

\section{References}

[1] Nouredine Zettili. Quantum mechanics : concepts and applications. Wiley, Chichester, U.K, 2009. 
[2] Paola Cappellaro. Quantum Theory of Radiation Interactions - Nuclear Science and Engineering MIT OpenCourseWare. https://ocw.mit.edu/courses/nuclear-engineering/, fall 2012. (Accessed on 06/25/2020).

[3] Raj Chakrabarti and Herschel Rabitz. Quantum control landscapes. International Reviews in Physical Chemistry, 26(4):671-735, 2007.

[4] Navin Khaneja, Timo Reiss, Cindie Kehlet, Thomas Schulte-Herbrüggen, and Steffen J Glaser. Optimal control of coupled spin dynamics: design of NMR pulse sequences by gradient ascent algorithms. Journal of magnetic resonance, 172(2):296-305, 2005.

[5] Shai Machnes, Elie Assémat, David J Tannor, and Frank K Wilhelm. Gradient optimization of analytic controls: the route to high accuracy quantum optimal control. arXiv preprint arXiv:1507.04261, 2015.

[6] T Schulte-Herbrüggen, A Spörl, N Khaneja, and SJ Glaser. Optimal control for generating quantum gates in open dissipative systems. Journal of Physics B: Atomic, Molecular and Optical Physics, 44(15):154013, 2011.

[7] Peter Lax. Linear algebra and its applications. Wiley-Interscience, Hoboken, N.J, 2007.

[8] Carl Wunsch. Discrete inverse and state estimation problems: with geophysical fluid applications. Cambridge University Press, 2006.

[9] Thomas Mehen. BCH.pdf. http://webhome.phy.duke.edu/ mehen/760/ ProblemSets/BCH.pdf, Jan 2009. (Accessed on 06/24/2020).

[10] Ralph M Wilcox. Exponential operators and parameter differentiation in quantum physics. Journal of Mathematical Physics, 8(4):962-982, 1967.

[11] quantum mechanics - Does a time-dependent Hamiltonian commute with its self at different times? https: / / physics.stackexchange.com, jan 2020. (Accessed on 1/15/2020).

[12] Wen-Xiu Ma, Xiang Gu, and Liang Gao. A note on exact solutions to linear differential equations by the matrix exponential. Adv. Appl. Math. Mech, 1(4):573-580, 2009.

[13] Wen-Xiu Ma and Boris Shekhtman. Do the chain rules for matrix functions hold without commutativity? Linear and Multilinear Algebra, 58(1):79-87, 2010.

[14] James H Liu. A remark on the chain rule for exponential matrix functions. The College Mathematics Journal, 34(2):141-143, 2003.

[15] operators - The formal solution of the time-dependent Schrödinger equation. https: / physics . stackexchange.com, jan 2020. (Accessed on 1/14/2020).

[16] Jay A Wood. The chain rule for matrix exponential functions. The College Mathematics Journal, 35(3):220-222, 2004.

[17] quantum field theory - Does the time ordering operator have a rigorous definition? https: // physics. stackexchange. com, jan 2020. (Accessed on 1/15/2020).

[18] Jearl Walker and Resnick Halliday. Fundamentals of physics. John Wiley \& Sons, Inc, Hoboken, NJ, 2014. 
[19] quantum mechanics - Time ordering operator and derivative with respect to time. https:// physics.stackexchange. com, jan 2020. (Accessed on 1/15/2020).

[20] quantum mechanics - Utility of the time-ordered exponential. https://physics . stackexchange. com, jan 2020. (Accessed on 1/15/2020). 